\title{
Balkanologie
}

Balkanologie Revue d'études pluridisciplinaires

Vol. II, nº 2 | 1998

Volume II Numéro 2

\section{La propagande politique}

L'exemple de la Bulgarie communiste à travers les marques et les logos

\section{Maria Tzvetkova}

\section{CpenEdition}

\section{Journals}

Édition électronique

URL : http://journals.openedition.org/balkanologie/259

DOI : 10.4000/balkanologie.259

ISSN : 1965-0582

Éditeur

Association française d'études sur les Balkans (Afebalk)

\section{Édition imprimée}

Date de publication : 1 décembre 1998

ISSN : 1279-7952

Référence électronique

Maria Tzvetkova, «La propagande politique », Balkanologie [En ligne], Vol. II, n² 2 | 1998, mis en ligne le 02 juin 2008, consulté le 17 décembre 2020. URL : http://journals.openedition.org/balkanologie/259 ; DOI : https://doi.org/10.4000/balkanologie.259

Ce document a été généré automatiquement le 17 décembre 2020.

(c) Tous droits réservés 


\title{
La propagande politique
}

L'exemple de la Bulgarie communiste à travers les marques et les logos

\author{
Maria Tzvetkova
}

\section{NOTE DE L'ÉDITEUR}

Cet article constitue un chapitre du mémoire Signes des idéologies: Marques industrielles et commerciales, logos, idéologies dans la Bulgarie communiste, réalisé sous la direction de Bernard Béney, soutenu en novembre 1996 (il est disponible sur demande auprès de son auteur).

\section{La propagande politique}

1 Les marques et logos, vecteurs d'images et de références, sont une contribution essentielle à la publicité des entreprises et des produits. Parallèlement,

l'homme politique qui veut conquérir le Pouvoir, ou qui veut le conserver, doit nécessairement être médiatique. C'est ainsi que [...] les conseillers en communication jouent un rôle de plus en plus important auprès des responsables politiques. Conduite à son terme, la logique médiatique fait de l'homme et du discours politiques un "produit" en tout point comparable à un autre, une "marchandise" qu'il s'agit de vendre en utilisant des méthodes éprouvées dans le domaine de la publicité et du commerce ${ }^{1}$.

2 C'est dans cette logique que le Parti Communiste Bulgare ( $\mathrm{PCB}$ ) au pouvoir, par exemple, a confié la réalisation des logos de ses congrès à la compétence de graphistes ou que, plus récemment, l'Union des Forces Démocratiques a contacté le publicitaire Jacques Séguéla pour mener sa campagne électorale de $1992^{2}$. Michel Bongrand parle d'ailleurs d'une correspondance entre marketing commercial et marketing politique à travers « le produit, le marché, le consommateur, la différence, la vente, le profit »3.

3 Pour Jean-Marie Domenach, « la propagande se rapproche de la publicité en ce qu'elle cherche à créer, transformer ou confirmer des opinions et qu'elle use en partie de moyens qu'elle lui a empruntés; elle s'en distingue en ce qu'elle vise un but politique et 
non commercial $»^{4}$ et "[la publicité] cherche à "frapper" plutôt qu'à convaincre, à suggestionner plutôt qu'à expliquer. Le slogan, la répétition, les images attrayantes l'emportent progressivement sur les annonces sérieuses et démonstratives $»^{5}$. Alors que pour Serge Tchakhotine, la publicité "cherche même à créer le besoin chez celui auquel elle s'adresse. Ce sont les mêmes règles techniques que [...] dans le dressage, seulement, comme l'on a affaire ici à des êtres humains, on utilise des systèmes de réflexes conditionnés d'un plan plus élevé, et naturellement, on joue sur toute la gamme des pulsions et de leurs dérivés $»^{6}$. Bref, pour Serge Tchakhotine, même si les buts sont divergents, les moyens utilisés par la publicité et la propagande politique sont du même ordre : ceux de la manipulation mentale.

4 Toutefois, nous ne sommes pas aussi catégoriques que Jean-Marie Domenach quant à savoir qui de la propagande politique ou de la publicité a emprunté les moyens et les techniques de l'autre. Mais il est troublant de constater que le développement des marques, des logos et de la réclame coïncide avec celui des techniques modernes de propagande politique, elle-même « liée à l'introduction dans l'histoire moderne des grandes idéologies politiques conquérantes (jacobinisme, marxisme, fascisme) et à l'affrontement de nations et blocs de nations dans les guerres nouvelles ${ }^{7}$.

Dans le cas de la propagande politique, les objectifs principaux de ceux qui l'utilisent sont généralement :

- la conquête du pouvoir,

- l'instauration d'un système économique ou politique particulier,

- le maintien au pouvoir,

- la pérennisation d'un système économique ou politique (ce qui n'empêche pas son évolution, comme le démontrent par exemple les changements sans rupture profonde survenus dans les pays communistes après la mort de Staline),

- l'extension géographique d'un pouvoir, d'un système économique ou politique.

6 Certains de ces objectifs peuvent être dissociés, mais pour beaucoup d'acteurs de la politique, la conquête du pouvoir, le maintien au pouvoir, l'instauration d'un nouveau régime et souvent l'extension géographique d'un système économique ou politique font partie d'un même objectif.

7 Or, théorisant différentes stratégies afin de conquérir le pouvoir et de s'y maintenir, Niccolo Machiavel reconnaît l'intérêt d'être soutenu dans ses ambitions par le plus grand nombre :

Si l'on considère la conduite des divers princes d'Italie qui, de notre temps, ont perdu leurs États (...), on trouvera d'abord une faute commune à leur reprocher, c'est celle des forces militaires (...). En second lieu, on reconnaîtra qu'ils s'étaient attirés la haine du peuple (...).

Philippe de Macédoine, non pas le père d'Alexandre le Grand, mais celui qui fut vaincu par T. Quintus Flaminius, ne possédait qu'un petit État en comparaison de la grandeur de la république romaine et de la Grèce, par qui il fut attaqué ; néanmoins, comme c'était un habile capitaine, et qu'il avait su s'attacher le peuple et s'assurer des grands, il se trouva en état de soutenir la guerre durant plusieurs années; et si, à la fin, il dut perdre quelques villes, du moins il conserva son royaume ${ }^{8}$.

C'est approximativement ce qu'en d'autres termes exprime Joseph Goebbels au Congrès de Nuremberg du 6 septembre 1934 : « il est peut-être bon de posséder la puissance qui repose sur la force des armes. Mais il est meilleur et plus durable de gagner le cœur d'un peuple et de le conserver ». 
Certes, Niccolo Machiavel et Joseph Goebbels ne conçoivent pas l'instauration de systèmes politiques où l'accession au pouvoir passe obligatoirement par des élections et donc par le soutien d'une majorité de votants. Toutefois, qu'il s'agisse d'instaurer une dictature ou un régime "démocratique", de mettre en place un système politique nouveau ou de le pérenniser, la réalisation de chacun de ces objectifs passe généralement par l'adhésion et le soutien du plus grand nombre.

10 La propagande s'avère alors être l'un des instruments les plus efficaces pour s'attacher ce soutien.

11 Si la propagande n'est pas un phénomène nouveau (des graffiti électoraux ont été retrouvés à Pompéi, par exemple), elle est pourtant perçue comme l'un des phénomènes dominants $\mathrm{du} \mathrm{XX}^{\text {ème }}$ siècle, à tel point que "sans elle, les grands bouleversements de notre époque : la révolution communiste et le fascisme, n'auraient pas été concevables. C'est en grande partie grâce à elle que Lénine a pu instaurer le bolchevisme ; c'est essentiellement à elle que Hitler dut ses victoires ${ }^{9}$.

12 Ces graffiti de Pompéi n'ont donc aucune commune mesure avec la propagande politique moderne qui prend la forme d'» une technique scientifique. C'est dire qu'elle exploite rationnellement les données d'une science, la psychopolitique, avec des méthodes et des moyens qui, eux aussi, ressortissent désormais au domaine de la science $\aleph^{10}$. Science dont l'efficacité peut se mesurer par le niveau d'aveuglement du sujet manipulé ; la perfection dans le domaine de la manipulation étant « qu'il ne le sait pas, qu'il le nierait même si on le lui prouvait, persuadé, dans sa légitime fierté, d'être un libre citoyen d'un pays parfaitement démocratique $»^{11}$.

Pourtant, la propagande est loin d'être une science exacte et objective :

Le règne de la Propagande au XX ${ }^{\text {ème }}$ siècle repose autant sur ces grandes campagnes d'opinion orchestrées avec l'aide des multiples instruments inventés par la science, qu'il est motivé par l'atmosphère générale d'irréalité dans laquelle baigne l'humanité. C'est dire que l'imprimé, le cinéma ou la radio, découvertes du monde moderne, ne font que renforcer, amplifier et étendre une action qui a toujours existé justement par le fait qu'elle est le corollaire inévitable de ces activités naturelles de l'homme que sont la pensée, la parole, ou la création artistique. ${ }^{12}$

14 En matière politique, tout fait social et tout système de communication entre les hommes peut servir à convaincre. C'est d'ailleurs ce que pense Lénine en 1902 lorsqu'il affirme que

notre tâche est d'utiliser toutes les manifestations de mécontentement quelles qu'elles soient, de glaner et de moudre jusqu'aux moindres parcelles de protestation embryonnaire. Sans compter que des millions et des millions de paysans travailleurs, de petits artisans et autres seraient toujours avides d'écouter la propagande d'un social-démocrate tant soit peu habile. ${ }^{13}$

15 Tout peut servir à la propagande. Ainsi, l'exploitation du fait social exprimé aussi par Karl Marx lorsqu'il déclare qu'» il faut rendre l'oppression réelle plus dure encore, en y ajoutant la conscience de l'oppression et rendre la honte plus honteuse encore en la livrant à la publicité » peut se faire au détour d'une conversation, par l'intermédiaire du cinéma (que Lénine considérait, en tant qu'outil de propagande, comme «le plus important de tous les arts $\left.\aleph^{14}\right)$, d'une chanson, d'une affiche, d'un tract ou encore du réseau Internet.

- la parole : la simple conversation, la rumeur, le discours politique relayé par la radio, 
la chanson...;

- l'imprimé : le journal, le livre, le tract...;

- le spectacle: le théâtre, le cinéma... Nous pouvons également inclure dans cette catégorie les grands événements mis en scène tels que les défilés du $1^{\mathrm{er}}$ mai dans les pays communistes ou du 14 Juillet en France ;

- l'image :

photos, caricatures et dessins satiriques - emblèmes et symboles - portraits des chefs. L'image est sans doute l'instrument le plus frappant et le plus efficace. Sa perception est immédiate et n'exige aucune peine. Accompagnée d'une brève légende, elle remplace avantageusement n'importe quel texte ou quel discours. C'est en elle que se résume de préférence la propagande ${ }^{15}$.

17 Toutes ces catégories peuvent se combiner et se compléter ${ }^{16}$, mais nous attarderons essentiellement notre étude sur la propagande idéologique dans la Bulgarie communiste (1944-1989), à travers deux de ses supports : les marques et les logos.

\section{L'idéologie dans les marques et les logos bulgares}

18 À partir du 9 septembre $1944^{17}$, les staliniens au pouvoir en Bulgarie éliminent presque toute opposition en l'espace d'environ quatre années. Le PCB vise alors l'instauration d'un nouveau régime et son maintien au pouvoir. Cependant, ce nouveau régime étant imposé par une force extérieure au pays (l'URSS), beaucoup lui reste à faire pour se rallier la population.

\section{Écrire une nouvelle « réalité »}

19 Mise à part la terreur qui constitue en elle-même un acte de propagande, «la force de la propagande totalitaire repose sur sa capacité à couper les masses du monde réel ${ }^{18}$. La terreur et le mirage de la nouvelle "réalité" se justifient alors mutuellement aux yeux de certains: «quand les fins sont grandes, l'humanité use d'une autre mesure et ne juge plus le "crime" comme tel, usât-il des plus effroyables moyens $»^{19}$.

Les moyens de créer cette nouvelle "réalité" passent alors obligatoirement par «la contre vérité non vérifiable, le mélange vrai-faux, la désinformation du vrai, la modification du contexte, l'estompement avec sa variante : les vérités sélectionnées, le commentaire appuyé, l'illustration, la généralisation, les parts inégales, les parts égales $\aleph^{20}$. Par exemple, la nouvelle morale supprime dans les marques toute sensualité ; l'homme ou la femme ne sont officiellement plus que des prolétaires luttant pour l'édification du socialisme. 
Entreprise industrielle d'État Bulgaria, confection, 28 avril 1996.

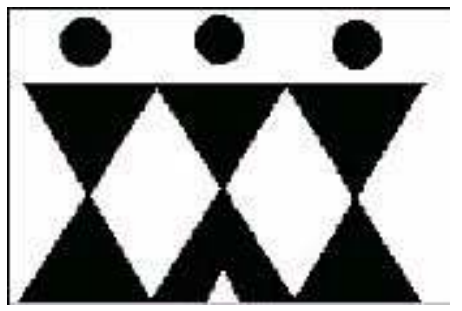

Afin de souligner l'égalité entre les sexe, l'homme et la femme, réduits à de simples silhouettes, sont disposés symétriquement côte à côte. Le nom de la maque associé au rythme répétitif dans une relation plus fraternelle que sensuelle, suggère la "vertu" du peuple dans le nouvel État bulgare.

Union commerciale d'État Valentina, chaîne de magasins de prêt-à-porter féminin, 1967.

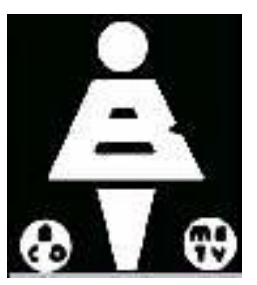

L'image de la femme est très rare dans les marques. Sa représentation est cependant l'occasion de mettre en avant son rôle de mère, procrétatrice d'une génération nouvelle. Notons que les canons de la beauté de l'époque (sveltesse) sont rejetés au profit de proportions et de formes proches d'une certaine Vénus de Willendorf.

Il s'agit pour le nouveau pouvoir d'éliminer méthodiquement toute référence à certains aspects du passé et de les remplacer afin de créer une nouvelle culture, en l'occurrence une culture monolithique de masse. Seule la version officielle a lieu d'exister. On élimine alors certaines notions (par exemple le confort et le luxe) que l'on remplace par l'expression de l'enthousiasme pour un avenir radieux construit par le prolétariat, forcément progressiste. Le code du progrès, surtout à l'époque du développement de l'industrie lourde, passe notamment par la représentation du boulon ou de la roue d'engrenage, entraînant sans aucun doute le progrès pour cet avenir radieux que suggèrent les nombreuses représentations du soleil.

Combinat de production Rodina (Patrie), Botevgrad, textile et cuir, $1^{\mathrm{er}}$ avril 1964.

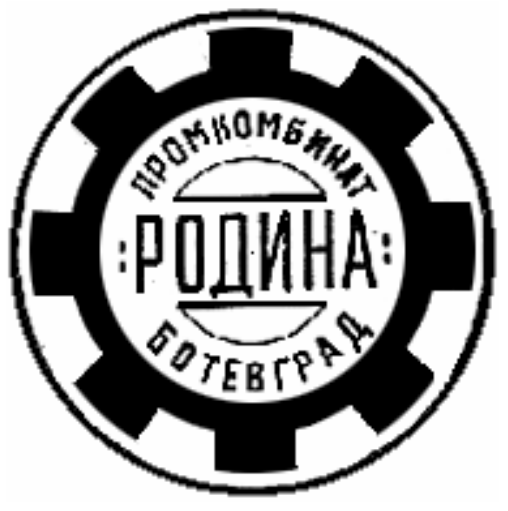


Machinoimport, Sofia, pièces de rechanges, 9 janvier 1964.

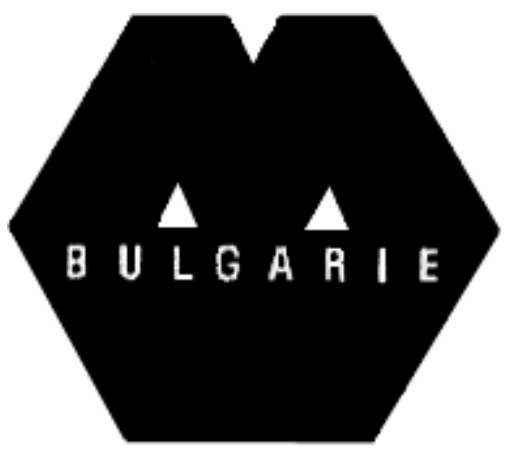

Entreprise de production socialiste Kiro-Konarov, Gorna Oriachovitza, production métallique, 1967.

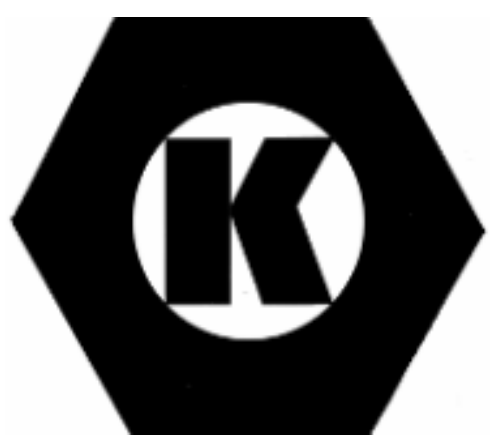

Entreprise industrielle d'État Beroé, production de machines, 1964.

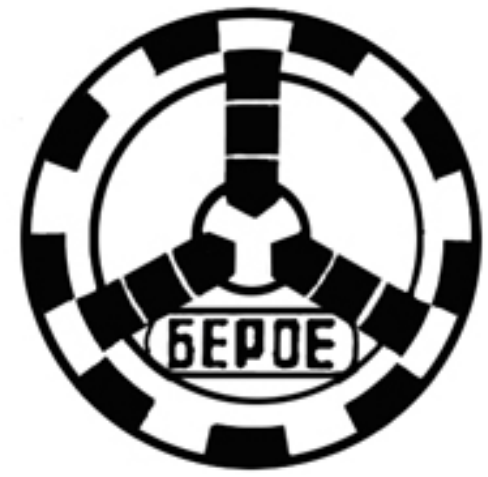

Tegko Mahinostroéné, construction de machines, Sofia, 26 avril 1968.

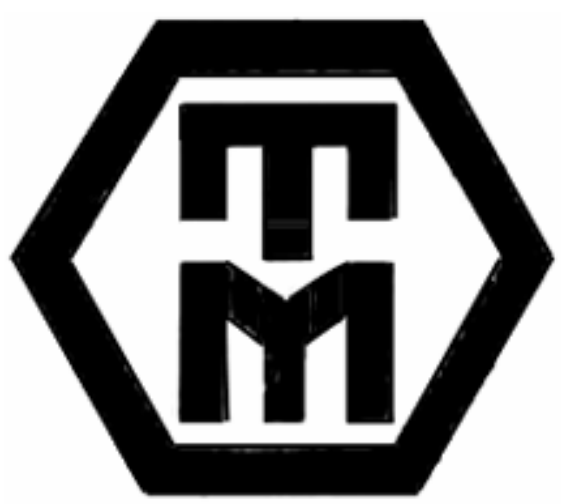


Coopérative de travail productif Izgrev (aurore), Plovdiv, production d'outils, 1969.

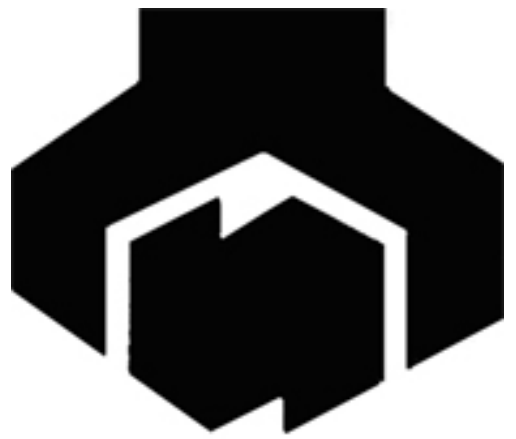

Pobeda (victoire), matériel électroménager, 1970.

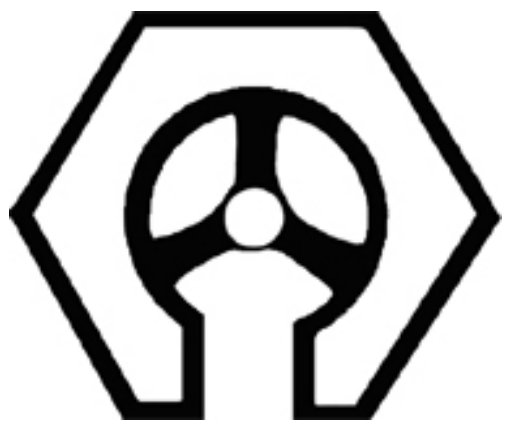

Coopérative de travail productif Samopomocht ("auto-aide"), Lom, confection de dessous féminins et de draps, 17 avril 1969.

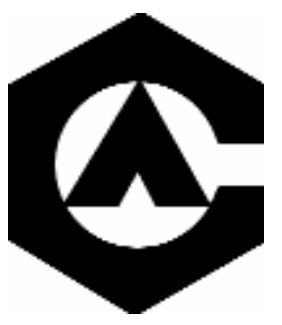

Machinoexport, Sofia, exportation de pièces de rechange, 25 novembre 1963.

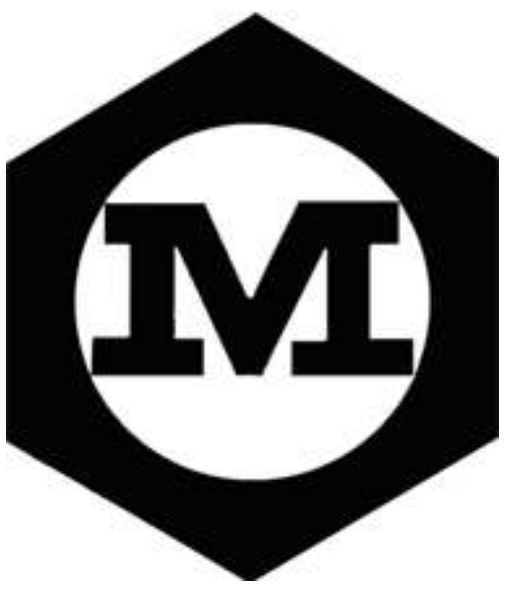


Rien n'arrête le progrès! Qu'il s'agisse de production de pièces de rechange, de cuir ou de dessous féminins, le boulon et la roue d'engrenage sont les constantes symboliques du progrès en marche

Fils Slantze (soleil) de l'Entreprise d'État Bulgaria, 1964

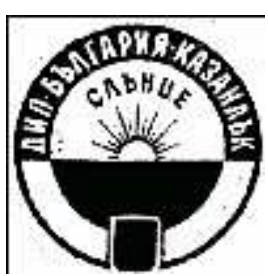

Les marques antérieures à 1944 portent généralement les noms de leurs anciens propriétaires ; les supprimer permet ainsi de rayer toute référence à la bourgeoisie. Mais c'est aussi mettre en application la vision totalitaire qui vise à remplacer l'individu par la masse :

l'individu ne fait pas partie d'un tout qui l'engloberait, mais - et c'est très différent - il est l'expression de ce tout. Ici, il n'y a plus d'individus, dans la mesure où il n'y a plus de parties, dans la mesure où le tout est partout. Pour atteindre ce but, et dans un premier temps, le totalitarisme atomise la société en détruisant toutes les structures traditionnelles qui sont génératrices d'idiosyncrasie. Puis, dans un deuxième temps, il coule comme dans un moule la matière informe, impersonnelle et anonyme des individus atomisés; dans cette perspective, les anciennes structures sont remplacées par de nouvelles qui ont non seulement pour but d'inculquer une idéologie, mais aussi de forger (au sens fort) des hommes nouveaux par identification à un type ${ }^{21}$.

Donc, mis à part l'utilisation rarissime de portraits de quelques personnalités (élevées au rang de mythes), toute référence à l'individu est abolie. À peine peut-on percevoir quelques silhouettes plus proches d'idéogrammes que de véritables personnes dans lesquelles tout Bulgare pourrait se reconnaître ${ }^{22}$. Les seules références à l'Homme sont incarnées par le rôle historique attribué à la masse : Prolétaire, Bolchevik, Héros.

\section{Étatiser l'Histoire et la mémoire}

L'une des premières mesures du nouveau pouvoir est alors de développer, au nom de la lutte des classes, la haine contre la bourgeoisie, et plus généralement contre les anciens propriétaires.

La première étape est la nationalisation des biens de ces bourgeois, l'élimination physique de quelques-uns et la déchéance (impossibilité d'accéder à l'université, de prendre part à la vie sociale, etc.) pour la grande majorité. La terreur pousse alors chacun à justifier de ses origines prolétaires (réelles ou supposées).

Une nouvelle "réalité" apparaît alors: avant 1944, la Bulgarie n'est composée exclusivement que de prolétaires opprimés par une infime minorité d'exploiteurs que le nouveau pouvoir a fini par empêcher de sévir. L'élimination de ces exploiteurs (entrave au développement) libère donc les énergies pour le progrès du pays.

Dans ce contexte, et avec le regroupement des anciennes entreprises par secteurs d'activité et la nationalisation, presque toutes les marques d'avant-guerre sont éliminées. 
Cette réécriture manichéenne de l'Histoire met donc en scène « les bons » et ses héros (le prolétariat) faisant disparaître les "méchants" (la bourgeoisie impérialiste et tout ce qui peut faire référence à elle). Les nouvelles marques ont donc pour noms Proletarii, Nezavissimost (indépendance), Tcherveno zname (drapeau rouge), Komsomoletz, GueorguiDimitrov, Dimitar-Blagoev ${ }^{23}$, Vassil-Kolarov ${ }^{24}$, etc.

Proletarii, Sofia, dessous et collants, 21 novembre 1966.

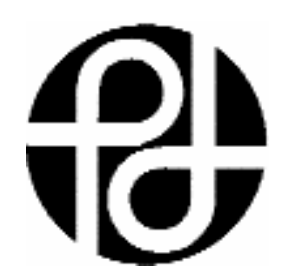

Bolchevik, Gabrovo, scies, 7 décembre 1964.

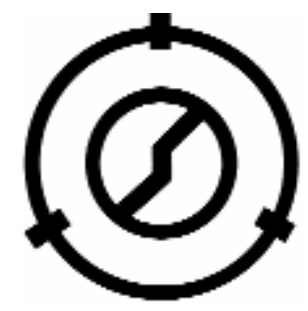

Komsomoletz, Sofia, confection, 8 avril 1969.

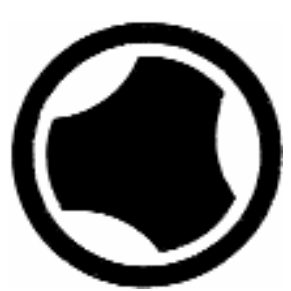

Mlada gvardia (jeune garde), Haskovo, traitement du tabac, 18 mars 1964.

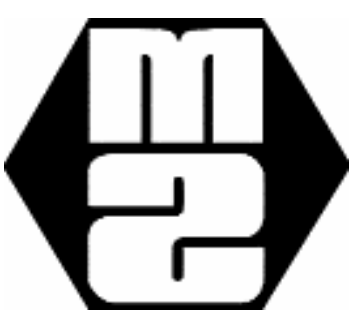

L'absence de figure d'un logo peut se retourner contre la propagande elle-même: concernant l'usine Proletarii, le logo s'est si profondément incrusté dans la conscience du consommateur bulgare qu'il en a fait oublier le nom de la marque. 
Usine d'État Tcherveno zname (drapeau rouge), machines agricoles, Stara Zagora, 29 janvier 1964.

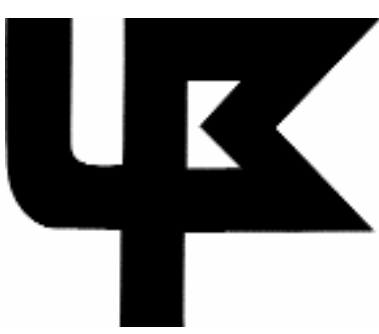

Toutefois, il est intéressant de constater que ces marques gardent la même conception et font appel aux mêmes références que les marques commerciales dans une économie libérale qui, selon Andrea Semprini, pour avoir un véritable impact auprès des consommateurs, doivent atteindre au moins l'un des trois niveaux de discours suivants :

Le niveau profond. - C'est le niveau [...] où quelques éléments seulement - abstraits et d'ordre général - se disposent en catégories d'opposés fondamentales et articulent les concepts fondateurs, les constantes socio-culturelles d'une société : vie et mort, bonheur et malheur, crime et châtiment, amour et haine, plaisir et répression.

Le niveau narratif. - À ce niveau, les constantes fondamentales sont mises en récit, organisées dans des suites d'action, construites selon la logique des scénarios. Ces scénarios restent abstraits, seuls quelques traits fondamentaux y sont esquissés : le héros, le méchant, l'être aimé... Mais c'est à ce niveau que se construit la composante narrative du discours de la marque.

Le niveau de surface. - Ce niveau correspond en partie à celui des signes. Les scénarios simplifiés du niveau précédent sont ici thématisés, mis en contexte, définis par tous les détails qui vont constituer leur spécificité et leur originalité. C'est à ce niveau que les quelques scénarios abstraits et génériques engendrent un nombre virtuellement infini d'histoires concrètes et spécifiques ${ }^{25}$.

Donc, « face à l'histoire telle qu'elle s'est déroulée, la propagande reconstitue l'histoire exemplaire, celle que les vainqueurs opposent aux vaincus, celle où ils se reconnaissent tels qu'ils souhaitent être vus ${ }^{26}$.

Nécessaire à la formation de "l'Homme nouveau", selon la terminologie officielle, l'enseignement de la nouvelle Histoire passe par un processus de réécriture permanente parfaitement décrit par George Orwell :

Lorsque toutes les corrections qu'il était nécessaire d'apporter à un numéro spécial du Times avaient été rassemblées et collationnées, le numéro était réimprimé. La copie originale était détruite et remplacée dans la collection par la copie corrigée. Ce processus de continuelle retouche était appliqué, non seulement aux journaux, mais aux livres, périodiques, pamphlets, affiches, prospectus, films, enregistrements sonores, caricatures, photographies. Il était appliqué à tous les genres imaginables de littérature ou de documentation qui pouvaient comporter quelque signification politique ou idéologique. Jour par jour, et presque minute par minute, le passé était mis à jour. On pouvait ainsi prouver, avec documents à l'appui, que les prédictions faites par le Parti s'étaient trouvées vérifiées. Aucune opinion, aucune information ne restait consignées, qui aurait pu se trouver en conflit avec les besoins du moment. L'Histoire toute entière était un palimpseste gratté et réécrit aussi souvent que c'était nécessaire. Le changement effectué, il n'aurait été possible en aucun cas de prouver qu'il y avait eu falsification ${ }^{27}$. 


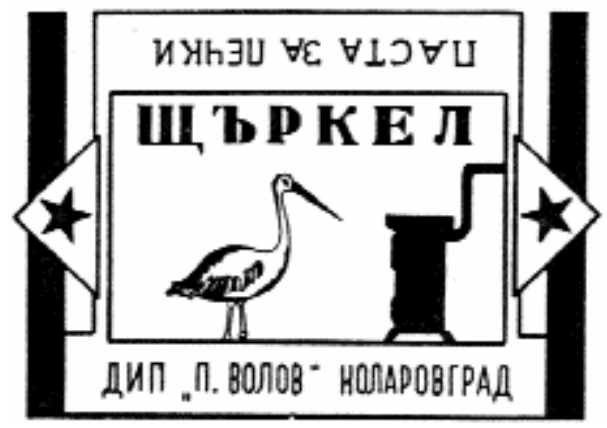

Les années 1950 ont consacré Vassil Kolarov, compagnon de lutte de Gueorgui Dimitrov, en lui attribuant le nom d'une ville (Kolarovgrad). Trop associée à la ville de Kolarovgrad par son logo (qui intègre le nom de la ville), cette marque est supprimée par l'État à l'époque de l'atténuation du culte de la personnalité, dans les années 1960. Peut-être est-ce aussi l'occasion d'éliminer le nom de l'une des dernières marques rescapées de l'avant-guerre...

La réécriture de l'Histoire ne touche donc pas seulement la période antérieure à 1944 mais aussi les disgraciés du régime en cours. « Aussi, la déstalinisation se bornait-elle outre des règlements de comptes personnels et quelques changements de noms et

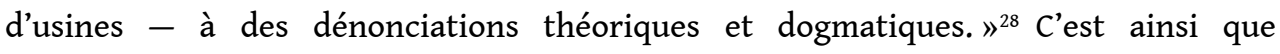
disparaissent les noms de Staline et de ses plus fervents partisans (comme Vassil Kolarov) des marques bulgares à la fin des années 1950 .

\section{Imposer la nouvelle culture}

La nouvelle culture est d'abord imposée par l'éducation (inculquant mensonges, contrevérités, vérités partielles et faisant carrément l'impasse sur des faits) ou par les médias qui réduisent l'information au niveau du simple message publicitaire.

Cette nouvelle culture fait notamment appel à certaines pulsions de l'individu répertoriées par Serge Tchakhotine dans les mécanismes de conservation:

Mécanismes de conservation de l'individu :

$\mathrm{N}^{\circ}$ I. Pulsion combative

$\mathrm{N}^{\circ}$ 2. Pulsion alimentaire.

Mécanismes de conservation de l'espèce :

$\mathrm{N}^{\circ} 3$ Pulsion sexuelle

$\mathrm{N}^{\circ} 4$ Pulsion parentale. ${ }^{29}$

Nous constatons à travers les marques et logos bulgares que la pulsion combative de l'individu, par exemple, est très fréquemment sollicitée. Pour Serge Tchakhotine, la « loi de la prépondérance du système combatif sur le système de la nutrition, régit les réflexes conditionnés des êtres supérieurs comme ceux des êtres les plus infimes [...]. Il faut retenir ce fait, parce que son importance sera mise en valeur dans le comportement des hommes à l'égard de la politique et de la propagande ${ }^{30}$. En effet, la combativité entretenue jusque dans les années 1970 à travers des marques comme Brigada, Boetz (combattant) ou Kolektiven troud (travail collectif) permet de maintenir une mobilisation permanente pour une cause, éventuellement au détriment de la satisfaction des besoins matériels. On retrouve là aussi l'une des causes de la disparition de la notion de luxe et de confort dans les marques. 
Combinat Brigada, Assenovgrad, conserves, 4 mai 1972.

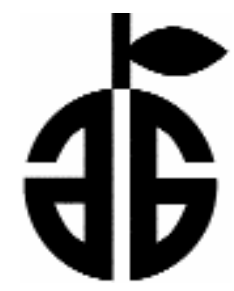

Coopérative de travail productif Kolektiven Troud (travail collectif), Pleven, chapeaux et casquettes, 16 avril 1969.

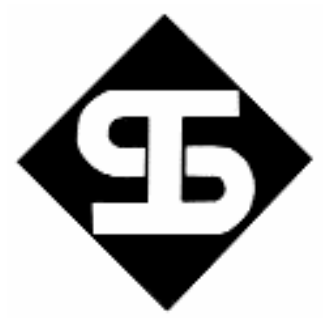

Coopérative de travail productif Boetz (combattant), Sofia, confection, 18 avril 1969.
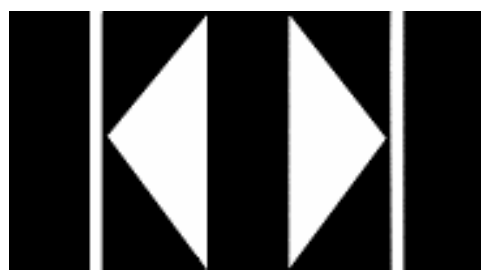

Usine Kibriti (allumettes), Kostenetz, 1978.

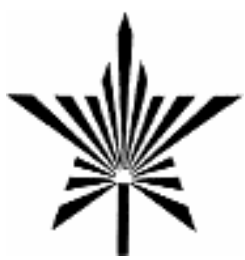

La mobilisation par le combat est l'un des fondements de la propagande du pouvoir entretenue, sous différentes formes, jusqu'à la fin du régime communiste (et même plus tard encore) : combat pour la quantité et la qualité de la production, pour la sécurité dans le travail, contre le fascisme, contre l'armement, pour la paix, etc.

Mais le principal objectif du nouveau pouvoir est avant tout de créer une culture de masse qui ne doit surtout pas être « créatrice, mais transmettrice et multiplicatrice ${ }^{31}$. Profitant de la mutation socio-économique du pays - faisant d'un peuple de paysans un peuple d'ouvriers -, le pouvoir cherche en fait à éliminer les cultures ancestrales pour leur substituer une culture homogène, de masse, se basant sur la tradition et la mémoire collective réécrites. Le but est alors de réunir et faire complaire la population dans cette nouvelle culture. 
Industrialimport, Sofia, import-export de textile, cuir, porcelaine, 30 décembre 1963.

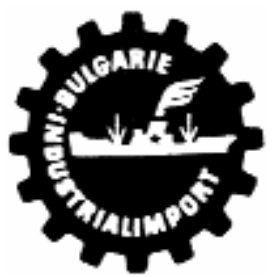

L'exportation (bateau) du progrès sans fin (roue d'engrenage), avec autant d'aisance que Mercure (aile), voilà l'un des mérites du socialisme (étoile).

Seuls les produits de qualité sont vendus à l'étranger ; n'est-ce pas alors aussi un peu de l'image du socialisme que l'on exporte? Et la reconnaissance des produits bulgares par l'étranger n'est-elle pas aussi la démonstration aux Bulgares eux-mêmes du bon fonctionnement du système?

Coopérative Zarneni Chrani, Sofia, céréales, 9 décembre 1967.

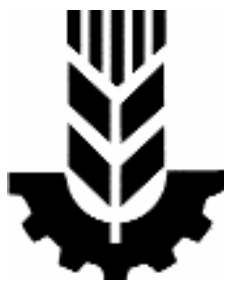

Ce logo reprend une partie des armoiries de l'État socialiste : l'union des paysans et des ouvriers par le blé et la roue d'engrenage.

Dans son contexte, cette symbolique participe à la réécriture de l'Histoire en suggérant que le progrès technique ne s'est réalisé qu'à partir de 1944, niant la mécanisation de la première moitié du XIXème siècle.

41 Imposée par l'État, le pouvoir veut cette culture à l'image de la nouvelle structure sociale. Le projet n'est alors peut-être pas si éloigné du Meilleur des mondes d'Aldous Huxley :

- La population optima, dit Mustapha Menier est sur le modèle de l'iceberg : huit neuvièmes au-dessous de la ligne de flottaison, un neuvième au-dessus.

- Et ils sont heureux, au-dessous de la flottaison?

- Plus heureux qu'au-dessus. Plus heureux que vos amis que voici, par exemple.- Il

les désigna du doigt.

- En dépit de ce travail affreux?

- Affreux? Ils ne le trouvent pas tel, eux. Au contraire, il leur plaît. Il est léger, il est d'une simplicité enfantine. Pas d'effort excessif de l'esprit ni des muscles. Sept heures et demie d'un travail léger, nullement épuisant, et ensuite la ration de soma, les sports, la copulation sans restriction, et le Cinéma Sentant. Que pourraient-ils demander de plus ? ${ }^{32}$

Le rôle attribué à la population est alors de reproduire les mêmes clichés intégrant une fois encore la nouvelle écriture de l'Histoire et de la mémoire, sachant que

l'idéologie de toute formation sociale n'a pas seulement pour fonction de refléter le processus économique, mais aussi de l'enraciner dans les structures psychiques des hommes de cette société. C'est à un double titre que les hommes sont tributaires de leur condition d'existence: ils en dépendent directement par ses incidences économiques et sociales, indirectement par l'intermédiaire de la structure idéologique de la société ; ils doivent donc développer toujours dans leur structure psychique une antinomie répondant à la contradiction entre les répercussions de leur situation matérielle et les répercussions de la structure idéologique de la société à laquelle ils appartiennent. Ainsi, l'ouvrier se trouve soumis tant aux influences de sa situation de travail qu'à celles de l'idéologie générale de la société. 
Comme les hommes faisant partie des différentes couches ne sont pas seulement les objets de ces influences mais les reproduisent aussi comme individus actifs, leur pensée et leur action doivent être aussi contradictoires que la société d'où elles émanent. Comme une idéologie sociale modifie la structure psychique des hommes, elle ne s'est pas seulement reproduite dans ces hommes, mais - ce qui est le plus important - elle a pris dans la forme de l'homme concrètement modifié et agissant d'une manière modifiée et contradictoire le caractère d'une force active, d'une puissance matérielle. ${ }^{33}$

\section{Le culte du chef} parentale répertoriée par Serge Tchakhotine et que l'on retrouve dans les termes "Père des peuples" (Staline) ou moins solennellement "Papa" (Todor Jivkov ${ }^{34}$ ), voire "Tonton" (François Mitterrand). Particulièrement "Dans les circonstances tragiques, cette projection sur le chef est favorisée par le besoin de chercher refuge auprès d'un "père" qui vous protège $»^{35}$, même s'il s'agit de l'oppresseur ${ }^{36}$.

Et « toute l'habileté de la propagande consiste à nous faire croire que cet homme d'État, ce chef de parti, ce gouvernement nous "représentent", non seulement défendent nos intérêts, mais assument nos passions, nos soucis, nos espoirs $»^{37}$ en légitimant aussi les actes par-delà la morale personnelle ${ }^{38}$.

\section{Entreprise d'État Vassil-Kolarov, 23 février 1953 et 26 janvier 1965.}

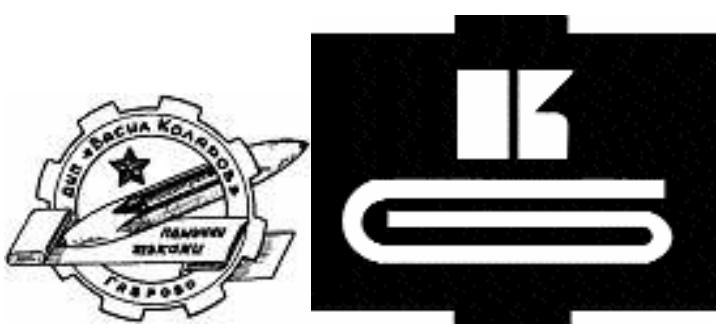

La nationalisation englobe dans cette marque une vingtaine de fabriques de textile de Gabrovo. Trop marqué du culte de la personnalité et de l'enthousiasme forcé des années staliniennes, le logo d'origine est remplacé par une variante moins connotée. Notons notamment la référence à Vassil Kolarov réduite à une simple initiale, plus anonyme...

La représentation de leaders, de chefs historiques et spirituels participe également à la réécriture de l'Histoire avec ses nouveaux héros présentés en modèle, mais aussi à fondre l'individu dans la masse :

Une multitude d'hommes devient une seule personne quand ces hommes sont représentés par un seul homme ou une seule personne, de telle sorte que cela se fasse avec le consentement de chaque individu singulier de cette multitude. Car c'est l'unité de celui qui représente, non l'unité du représenté, qui rend une la personne [Person]. Et c'est celui qui représente qui assume la personnalité [Person], et il n'en assume qu'une seule. On ne saurait concevoir l'unité d'une multitude, sous une autre forme. ${ }^{39}$

À la fin des années 1940, des opposants au nouveau régime tentent par tous les moyens d'interpeller le monde sur le système qui est en train de se mettre en place dans le pays et qui va perdurer jusqu'à la fin des années 1970. Écoutons par exemple les militants de la FACB (Fédération des anarchistes communistes de Bulgarie) ${ }^{40}$ concernant le culte de la personnalité :

Dans l'éducation sociale, les staliniens suivent le même principe et tendent au même objectif : servir les intérêts du parti et élever le culte des chefs. 
On se rappelle bien les thèses soutenues autrefois par les disciples de Marx et Engels, au sujet des facteurs historiques, surtout dans leurs discussions avec les anarchistes. Ils disaient et répétaient inlassablement leurs formules et leurs dogmes, expliquant le processus historique dans lequel aucun rôle n'était réservé à l'individu en tant que facteur conscient et agissant selon ses intérêts matériels et spirituels, ses convictions, ses idées et sa volonté. Il n'y avait alors que les forces économiques comme base matérielle et les masses prolétaires comme facteur unique et décisif pour transformer la société humaine.

Depuis ce temps-là, beaucoup de choses ont changé. On se souvient par exemple de tous ces procès politiques en Russie contre certains "facteurs" de l'opposition bolchevique, qui n'avaient pas jusqu'alors été reconnus tellement importants par le "matérialisme historique" et qui, cependant, auraient joué, paraît-il, un rôle décisif (néfaste cette fois) pour le développement historique et politique de l'U.R.S.S. (...) Dès lors, une nouvelle ère s'est ouverte, celle des chefs, des führers, des "pères des peuples", des "sages et géniaux conducteurs", des maréchaux, des généralissimes, etc., etc., qui, en réalité, sont devenus les vrais facteurs historiques, donnant et changeant la direction de tout le processus historique à une échelle mondiale. C'est justement le culte des chefs qui l'emporte, en se substituant au culte des masses. Aujourd'hui, on ne parle presque que des premiers ; les masses n'existent que pour servir de décoration, de place d'armes, de cadre historique aux grands gladiateurs de l'ère stalinienne.

À dire vrai, les staliniens continuent à ne reconnaître aucun rôle à l'individu, excepté, bien entendu, à l'individu-chef, dont le rôle est décisif cette fois. Et aujourd'hui, avec la même insistance et ténacité avec lesquelles ils avaient prêché autrefois les dogmes du vieux testament [de Marx-Engels-Lénine], ils disent et répètent les révélations des nouveaux prophètes.

Alors, il est tout naturel que le même changement s'opère dans le domaine de l'éducation des masses. En effet, nous voyons que le culte des chefs (ou mieux dire, du chef) occupe déjà la place unique dans l'emploi de tous les moyens pour... le bourrage de crâne.

La presse, la radio, le cinéma, l'art, la science, les syndicats, toutes les organisations professionnelles et leurs innombrables réunions, tous les meetings interminables, tous les discours, toutes les déclarations, ne chantent que l'éloge du chef avec les qualificatifs que tout le monde sait par cœur. C'est une chanson tellement connue et tellement ennuyeuse qu'on peut savoir au préalable le texte de chaque article de presse, de chaque discours de réunion, de chaque conférence, de chaque manifestation faite par n'importe lequel de tous les moyens de propagande stalinienne. ${ }^{41}$

Dans les années 1950, une énorme partie des marques vénère donc les leaders spirituels et historiques (Karl Marx, Lénine, Staline, etc.), tandis que les années 1960 à 1980 consacrent davantage des leaders nationaux tels que Dimitar Blagoev, Gueorgui Dimitrov $^{42}$, Angel Kantchev, Gueorgui Kirkov, Penka Mikhaïlova ou Ludmila Jivkova ${ }^{43}$.

Entreprise d'État Gueorgui-Dimitrov, pâtes alimentaires, Tcherven Briag, 22 janvier 1968.

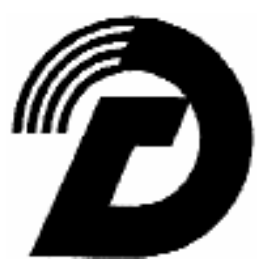




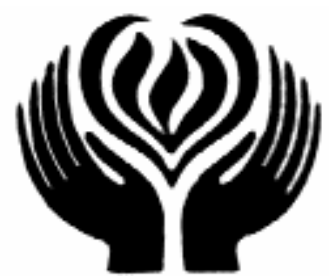

Tout comme le Palais de la culture de Sofia auquel le pouvoir attribue le nom de l'ancien ministre de la Culture (décédée en 1981), cette fondation est une expression tardive du culte de la personnalité. Le logo est cependant remarquable pour son évocation pertinente de l'engagement pour la culture auquel Ludmila Jivkova a consacré sa vie. Le logo intègre notamment le titre de son livre autobiographique : Pensez à moi comme à une flamme.

Mais, dans le cas très particulier des logos, ce qui caractérise l'utilisation de ces personnages historiques ou de ces dirigeants en place, c'est bien le manque d'incarnation visuelle (mis à part quelques cas extrêmement rares comme celui de Batcho Kiro). De la même façon que les anarchistes de la FACB qui utilisent ironiquement les termes religieux de "culte", "vieux testament" et "nouveaux prophètes", on ne peut manquer de constater une analogie entre l'absence totale de représentation visuelle des dirigeants et l'interdiction que ce font certaines religions de représenter leurs dieux (telles que les religions chrétienne ou musulmane).

«Remplacer le ciel »44, comme l'écrit l'auteur Ismaïl Kadaré, est même l'une des tâches du nouveau pouvoir communiste. Cette affirmation concernant l'Albanie est tout aussi valable pour la Bulgarie. Ainsi, durant toute l'époque communiste, après Staline, la Sainte Trinité est incarnée par Marx, Lénine et Dimitrov, accompagnée de toute une pléiade de saints et martyrs :

Sur l'autel des grandes figurations c'est le martyr qu'on révère, degré suprême de l'incarnation, identité d'une valeur et d'une vie où la mort identifie la personne à la cause. Figures de proue à innombrables variations historiques ou géographiques, conjuguant les images de la douleur, du sang et de la mort, ceux que l'événement a fixés dans la mort prennent leur revanche en orientant les conduites des vivants. ${ }^{45}$

Et d'ailleurs, peu importe les martyrs eux-mêmes, l'important étant avant tout de créer le mythe autour de leurs noms ; une fois le mythe créé (le concept, comme diraient les publicitaires), le nom est digne de faire partie du martyrologe. On trouve alors l'usine de bonbons "Maltchika", du surnom d'un étudiant communiste torturé à mort par la police pendant la guerre ou encore la fabrique de pâtes alimentaires "PenkaMikhaïlova", militante communiste tuée pour son idéal.

Entreprise d'État Penka-Mikhaïlova, pâtes alimentaires, Sofia, 10 mai 1968.

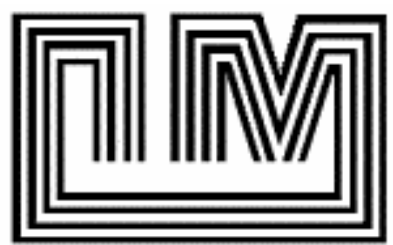




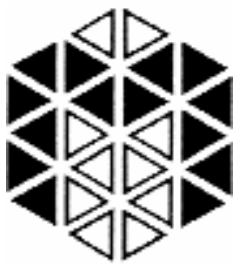

51 Poursuivant sur ce thème, on peut constater que graphiquement « les formes adoptées par les Églises pour leur propagande émotive, sont en principe absolument les mêmes que celles des mouvements politiques. Voyons, par exemple, les symboles : la Croix, comme symbole graphique, agit, en principe, de la même manière que [...] la faucille et le marteau des communistes $»^{46}$.

52 Tout comme les religions, le pouvoir se dote également d'événements symboliques à vénérer tels que le $1^{\mathrm{er}}$ mai ou le 9 sptembre (date officielle de la prise du pouvoir par les communistes). Or,

il n'y a pas de célébration sans icônes et la symbolique du Premier Mai s'apparente à une galerie de tableaux, une section d'iconologie comparée du musée imaginaire à la gloire du travail où annuellement chaque pays, chaque organisation mobilise ses artistes pour enrichir la collection d'une contribution supplémentaire. (...) C'est encore là la marque d'un sentiment religieux : provoquer l'exaltation de la foule par l'assomption du symbole, glorifier la référence pour susciter la révérence. ${ }^{47}$

53 Très souvent associés aux événements vénérés, les lieux sont aussi objets de culte : ordinateurs Pravetz ${ }^{48}$, Combinat textile d'État Maritza, agences de tourisme Pirin ${ }^{49}$ et Shipka ${ }^{50}$.

Matériel informatique Pravetz, 1986 et armoiries de la ville de Pravetz, 1984.

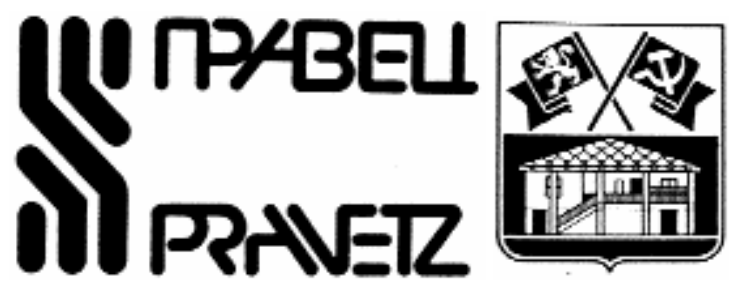

Par un effet secondaire du culte de la personnalité, Pravetz, ville natale du chef d'État Todor Jivkov, est élevée au rang de lieu mythique. Les armoiries de la ville vont jusqu'à consacrer la maison natale de Todor Jivkov en intégrant la symbolique en vigueur à l'époque : lieu mythique, culte de la personnalité, nationalisme (lion), socialisme, ...

Entreprise d'État 7-Maï, cuir, 1966, textile, 1965.

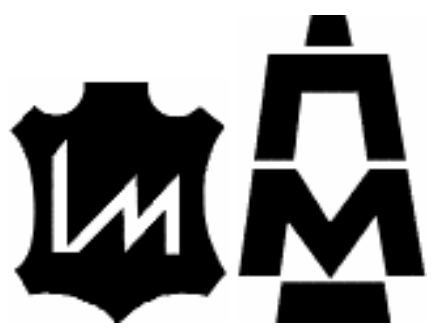


Entreprise d'État 9-Septemvri, cuir, 1965, chaussures, 1964.

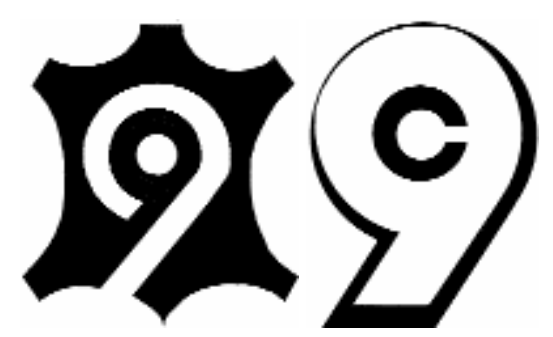

Comité du Parti Communiste pour la commémoration du 9 septembre, 1978.

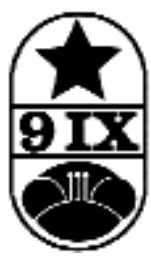

54 Mais ces lieux, ainsi que la référence aux héros de l'Histoire (particulièrement de l'indépendance) contribuent à affermir le pouvoir, tout en exaltant et entretenant le nationalisme.

Entreprise d'État Maritza, textile, 1978.

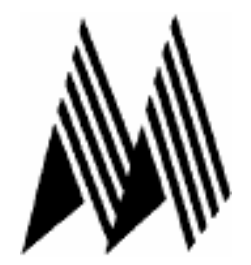

Agence de voyage Pirin, 1964.

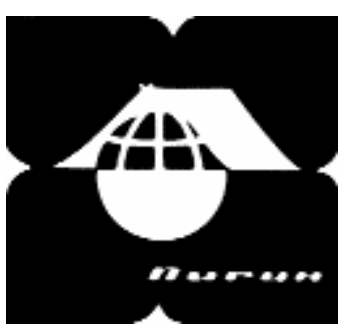

Association bulgare pour le tourisme, 1978.

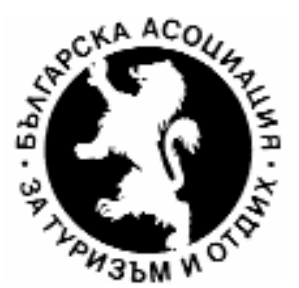




\section{Le nationalisme}

La nation n'est pas une réalité concrète, mais une idée. Elle n'est pas du même ordre que les formations sociales primaires telles que les clans, les tribus, les villages et les cités. Aucun des facteurs qui expliquent la formation de ces groupements, l'ethnie, le territoire, la religion, la langue, ne suffit à rendre compte de la réalité nationale. (...)

[Mais] en qualifiant (...) l'idée nationale, on n'a pas à s'interroger sur son origine et sa raison d'être. Elle fait partie de cet univers magique qu'est l'univers politique, dont les éléments ne sont pas des données objectives, mais des représentations et des croyances. À ce titre, la nation remplit deux fonctions essentielles dont l'exercice commande la survie des collectivités humaines: une fonction d'intégration qui procure au groupe la cohésion spirituelle grâce à laquelle il résiste à l'effet corrosif des rivalités d'intérêts; une fonction disciplinaire qui, en sacralisant le pouvoir, fait de sa force une autorité. (...)

[Or], la nation doit, sous peine de disparaître, parfaire sans cesse l'image qu'elle veut donner d'elle même ${ }^{51}$.

Le symbolique constitue alors l'une des meilleures expressions de son irrationalité : "c'est sur la visée d'une identité singulière à construire que repose l'efficacité du symbolique: une communauté, par exemple, cherche à se définir par des traits distincts, et pour cela cherche à se constituer une mémoire, une histoire, des images. Sans cette visée qui réunifie ses éléments hétérogènes, groupe, communauté ou société n'ont pas d'existence symbolique $»^{52}$.

Si l'on aborde la thématique nationaliste, «il semble permis de constater l'existence d'un fonds idéologique commun, organisé autour de quatre thèmes principaux: souveraineté, unité, passé historique et prétention à l'universalité $»^{53}$. Ces thèmes touchent d'ailleurs tous les domaines, de la production à l'art, en passant par la science et les loisirs (comme l'attestent les marques des agences de voyage ou des clubs de sport à forte symbolique nationaliste).

La souveraineté de la nouvelle nation socialiste se construit d'abord autour de la symbolique du Parti communiste. Quasiment groupusculaire juste avant la guerre, ce parti s'affirme en 1944 avec l'appui de l'URSS et impose à la société entière sa symbolique socialiste. Dans les années 1960, les liens entre l'État et le Parti communiste étant devenus indissociables à tous les niveaux (économiques, juridiques, artistiques, etc.), l'affirmation de la souveraineté de l'État-Parti s'exprime alors par sa volonté de reconnaissance internationale, notamment à travers les marques d'exportation où prédomine la symbolique politique.

Maison d'édition du Parti communiste Partizdat, 1969.

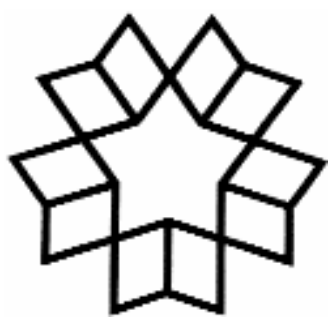

L'étoile, constituée par les livres, s'affirme néanmoins comme leur point central. Ce symbolisme ne matérialise-t-il pas graphiquement le slogan du chef de l'État Todor Jivkov : « une démocratie populaire pour former une nation démocratique »?

Dans les faits, le symbole de l'étoile au cœur de la pensée reflète, autant par l'édition que par sa censure, la conception de la diffusion du savoir selon l'État. 
Ne doutant plus de sa souveraineté dans les années 1980, la reconnaissance par le monde de la nationalité bulgare s'exprime alors surtout à travers la propagation d'images suggérant l'internationalisme (telles les images des nombreux festivals culturels internationaux).

Commémoration de 90 ans de presse politique (sous-entendu communiste), 1982.

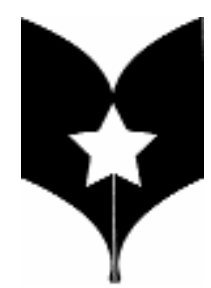

Comité du Parti communiste pour la commémoration du quarantième anniversaire de la Révolution socialiste en Bulgarie, 1984.

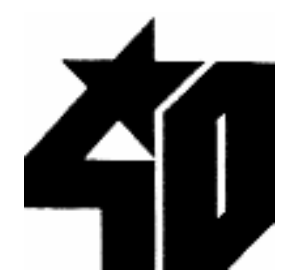

59 Aussi, la souveraineté nationale se traduit à travers les grandes luttes pour cette souveraineté elle même. Des marques portent alors des noms mythiques comme Shipka, faisant référence à une bataille capitale pour l'indépendance du pays...

L'unité, quant à elle, se crée autour de valeurs politiques et sociales. Le travail, par exemple, présenté comme essentiel dans la société socialiste, est utilisé comme référence commune aux citoyens de toute culture (Bulgares, $\operatorname{Turcs}^{54}$, Tsiganes, etc.).

La suppression officielle des classes sociales constitue aussi un élément unificateur, l'ensemble des individus étant censé se retrouver à travers la marque Prolétaire. Mais peut-être plus que par l'application de sa politique, c'est le Parti communiste lui-même qui, par son omniprésence, se présente comme l'élément unificateur dans l'État-nation. C'est d'ailleurs cette conception de la société qu'exprime dès 1917 Joseph Staline à propos de "la question nationale":

Il y a un plan d'après lequel les ouvriers s'organisent par nationalités : autant de nations, autant de partis. Ce plan a été repoussé par la social-démocratie. La pratique a montré que l'organisation par nationalités du prolétariat d'un État donné ne fait qu'étouffer l'idée de solidarité de classe. Tous les prolétaires de toutes les nations composant un État donné doivent être organisés en une collectivité prolétarienne une et indivisible..$^{55}$

Mais l'unité se crée aussi dans la différence. Ainsi, dans les années 1960, le Parti communiste réhabilite les "intellectuels" qu'il avait jusqu'alors dénigrés à travers sa propagande en les opposant à ceux qui produisent des biens "utiles" pour tous, c'est-àdire matériels. Au même titre qu'ouvrier et paysan, on peut être maintenant "ouvrier sur le front culturel”, termes qui permettent de reconnaître la valeur des universitaires ou des artistes, par exemple, sans pour autant remettre en question des années de propagande. 
63 La création d'unions professionnelles peut alors s'assimiler, par un caractère corporatiste, à une forme de nationalisme basé sur des groupes sociaux identitaires : unions des agriculteurs, des architectes, des graphistes, etc. Chaque union distincte constituant un élément présenté comme complémentaire aux autres et donc indispensable au fonctionnement de la société, c'est de la différence et de la spécificité que se crée l'unité.

Troisième concours international des jeunes cuisiniers, pâtissiers et serveurs, Varna, 1972.

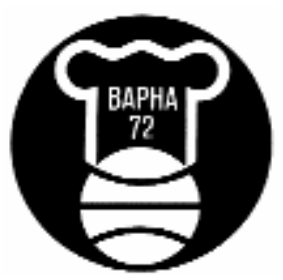

Union des écrivains maritimes, Burgas, 1978.

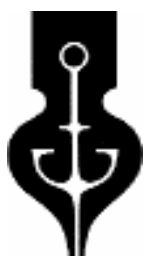

Union des architectes des pays balkaniques, 1978.

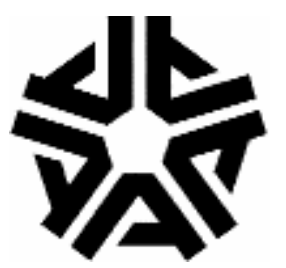

Union des juristes du droit maritime, 1982.

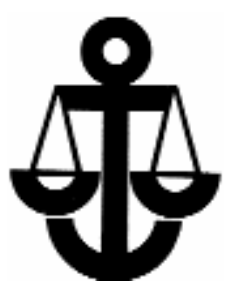

Association Généraux pour la paix et le désarmement, 1988.

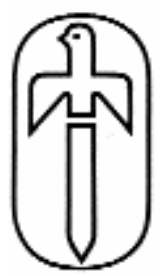


64 L'unité de la nation bulgare se crée donc autour de valeurs politiques nouvelles, ce qui n'empêche pas de faire réapparaître dans les années 1970 les images fortes de la symbolique nationaliste bulgare "traditionnelle", comme le lion ou la rose... Les valeurs du socialisme et de ses symboles perdant de leur puissance mobilisatrice, il s'agit en fait de tenter de souder des liens nationaux à travers l'imagerie traditionnelle. Mais le calcul du pouvoir utilise également l'idée de mobilisation autour d'un ennemi : le Turc, qui a l'“avantage" en l'occurrence d'être à la fois “ennemi" intérieur et extérieur.

Usine Dianko-Stephanov, production de verre, 1982.

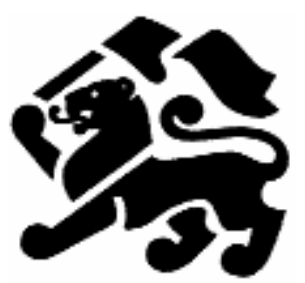

Car contrairement à la mobilisation unitaire autour des valeurs socialistes, l'imagerie traditionnelle bulgare a la particularité d'exclure de la nation les minorités telles que les Turcs. La plaie de l'Histoire de nouveau ouverte, dans les années 1980,

À côté des manifestations glorifiant le passé bulgare, le PC déclenche la phase finale d'une opération commencée au cours des décennies précédentes sur une moindre échelle. Il s'agit de l'“édification d'une nation bulgare unie", débarrassée de toute notion de minorité. (...) Les autorités annoncent que tous les Turcs vivant en Bulgarie sont en réalité des descendants de Bulgares islamisés de force sous le joug turc et que, désormais, "ils demandent tous, spontanément, la restauration de leurs noms bulgares". En pratique, cette "restauration" se déroule comme une opération militaire de grande envergure. (...) [Les Turcs] n'ont plus le droit, sous peine d'amende ou d'autres sanctions administratives, de porter des vêtements traditionnels turcs, ni de parler entre eux la langue turque dans un lieu public. ${ }^{56}$

Quant à ceux qui refusent de se soumettre à la "bulgarisation" de leurs noms, ils sont poussés à l'exode vers la Turquie par centaines de milliers à partir de $1984 . .$.

Dans un tel contexte, il n'est donc pas surprenant qu'aucune spécificité culturelle turque ne soit perceptible à travers les marques et les logos...

Comme nous l'avons vu à propos de la réécriture de l'Histoire, la maîtrise du passé est capitale pour le pouvoir. Or, dans une perspective nationaliste, cette maîtrise passe par la récupération du temps et de l'espace.

69 La récupération du temps vise à retrouver une sorte de paradis perdu incarné par exemple par les anciens royaumes. Ainsi, par une inversion symbolique, le lion des armoiries de la République populaire de Bulgarie s'appuyant sur la roue d'engrenage suggère aux Bulgares l'époque glorieuse de la conquête de l'indépendance, lorsque le lion foulait du pied le croissant des Ottomans.

70 Mais si le symbole du lion marchant sur le croissant est évidemment perçu de façon différente (voire contraire) par les Bulgares et les Turcs de Bulgarie, l'unité nationale se fait autour de la roue d'engrenage, symbole du progrès et du travail... 
Broderie et drapeau des insurgés bulgares, 1876. À l'époque où le lion bulgare foule du pied d'Empire ottoman symbolisé par le croissant.
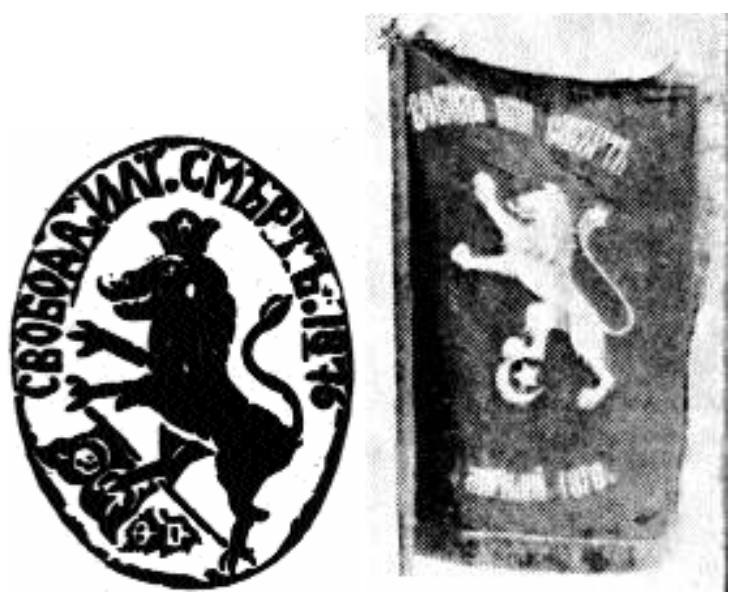

Armoiries de la République populaire de Bulgarie, de 1944 à 1992.

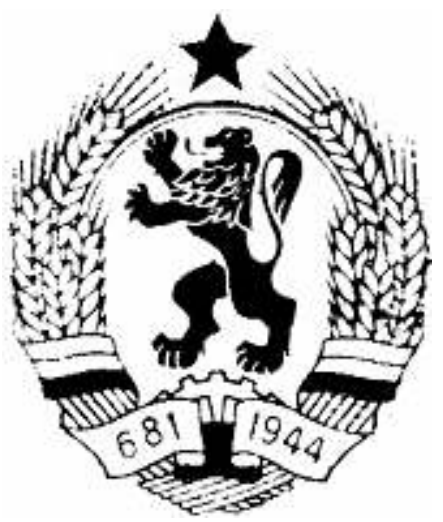

En se tournant vers le passé en attribuant aux marques des noms de lieux historiques, il est aussi question de faire revivre un sentiment nationaliste par le rappel d'événements présentés comme essentiels à l'Histoire et donc l'identité de la nation bulgare. Cela fait appel à une mémoire collective que le pouvoir tente de contrôler, éventuellement au prix de la réécriture des faits et des symboles.

L'image du passé constitue donc l'une des meilleures garanties d'avenir du pouvoir ; et c'est bien pour cela qu'il l'offre à la vénération :

L'idée nationale est le plus sûr élément du consensus; elle porte en elle une représentation du futur dont le pouvoir apparaît le garant. Sans doute, le souvenir des épreuves communes, les traditions, la conscience d'une originalité historique font la nation : mais, si les membres du groupe y sont attachés, c'est moins par ce que ces croyances représentent du passé que par ce qu'elles préfigurent de l'avenir. La nation, c'est continuer à être ce que l'on a été, à vivre selon la même foi que celle dont se sont inspirées les générations précédentes; c'est donc, à travers une image d'un passé, la vision d'un destin. "L'esprit, a écrit André Malraux, donne l'idée d'une nation: mais ce qui fait sa force sentimentale, c'est la communauté de rêve” ( $L a$ Tentation de l'Occident, 1926). De ce rêve, le pouvoir, en l'incarnant, fait un projet, d'où hier n'est évoqué que comme fondement de ce que sera demain. Dans cette perspective, le pouvoir est l'instrument de l'hypothèque prise sur l'avenir. ${ }^{57}$

De plus, l'utilisation de noms de territoires perdus (Prespa par exemple, du nom du lac partagé actuellement entre la Macédoine, l'Albanie et la Grèce) permet de revendiquer 
en quelque sorte leur appartenance à la nation Bulgare. Il en est de même avec la marque Dobrudja ${ }^{58}$ qui confirme son appartenance à la nation.

Entreprise d'État Raïna-Kniaginia, confection, 1978.



Raïna Kniaginia est l'héroïne à qui l'on atttribue la création du drapeau de la Bulgarie indépendante (1876). La logique nationaliste pousse alors le graphiste contemporain à renforcer la symbolique par le lion.

74 La prétention à l'universalité s'exprime, quant à elle, par la revendication de valeurs morales exportables et applicables au reste du monde ou tout au moins dans un environnement géographique le plus large possible.

Fédération sportive bulgare, 1978.

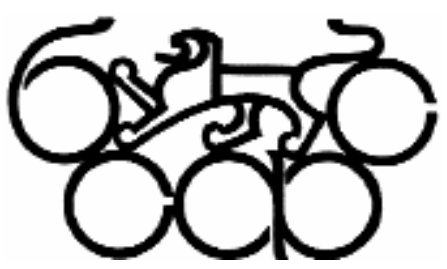

Le sigle disposé en analogie avec les anneaux olympiques, surmonté du lion, inscrit la Bulgarie dans un contexte international. C'est l'affirmation de la reconnaissance de la nation bulgare par le reste du monde.

Ainsi, sur le même principe que la France auto-proclamée "le pays des Droits de l'homme", le pouvoir de Sofia suggère dans les années 1980 une image idéalisée de l'enfance heureuse en Bulgarie, élevée au rang de modèle universel à travers l'assemblée internationale d'enfants "Drapeau de la paix".

Assemblée internationale d'enfants Drapeau de la paix, Sofia, 1980.

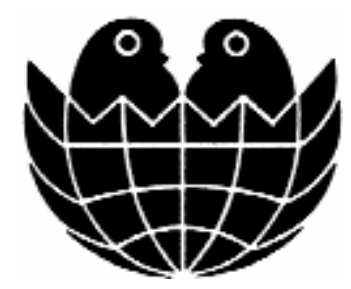

Douzième rencontre de la jeunesse des capitales socialistes, Sofia, 1983.

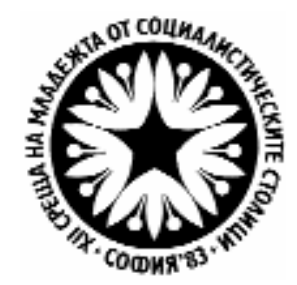






Entreprise économique Mladost (jeunesse), habillement et matériel de sport, 1980.

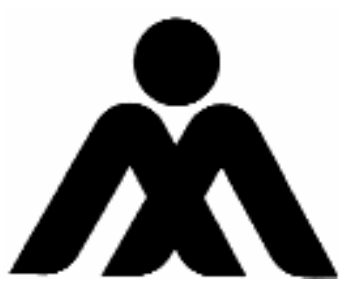

Derrière cette marque, l'État-Parti cherche à montrer sa préoccupation envers la jeunesse.

L'universalité se proclame également par l'affirmation forte de l'origine bulgare des caractères cyrilliques (bien que les frères Cyrille et Méthode soient reconnus d'origine grecque) tendant à présenter la nation bulgare comme un pôle culturel essentiel pour les centaines de millions d'humains utilisant cet alphabet.

Sous le couvert de la fête des saints Cyrille et Méthode (le 24 mai), le pouvoir va jusqu'à consacrer un Jour de l'Alphabet par une fête nationale, car l'alphabet cyrillique est dans les Balkans une façon de se démarquer des Grecs ou des Turcs et d'utiliser indirectement la religion orthodoxe comme facteur d'unification des slaves (en opposition à la Turquie majoritairement musulmane) :

La mission des frères Cyrille et Méthode en Moravie, au milieu du IX ${ }^{\text {ème }}$ siècle, avait provoqué chez les Slaves une prise de conscience de leur unité. La création d'un alphabet et d'une langue liturgique, communs à l'ensemble des Slaves du Sud et de l'Est, fut doublée par la mise au point d'une littérature de traduction, qui put être utilisée par tous les Slaves convertis par Byzance..$^{59}$

S'il s'agit alors de revendiquer l'origine de l'alphabétisation d'une partie de l'Europe et de l'Asie, c'est aussi la réaffirmation de liens historiques entre la Bulgarie et la Russie devenue Union soviétique...

\section{La contestation dans les marques}

79 Si l'on fait abstraction de tous les projets relégués dans les cartons par la censure, la contestation à travers les marques et logos est observable quasiment dès l'origine du pouvoir communiste.

On note par exemple une tendance à chercher à atténuer la propagande par la mise en avant graphique de la production au détriment de la symbolique socialiste; cette atténuation pouvant par exemple aller jusqu'à l'abandon dans le graphisme de toute référence au nom de la marque lié à l'un des "héros socialistes". 


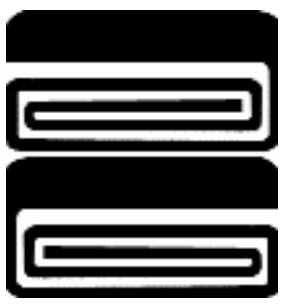

Le graphiste réussit à éclipser le nom du fondateur du Parti communiste bulgare en mettant en avant la symbolique de la production.

Entreprise d'État 9-Septemvri, chaussures, 1969.

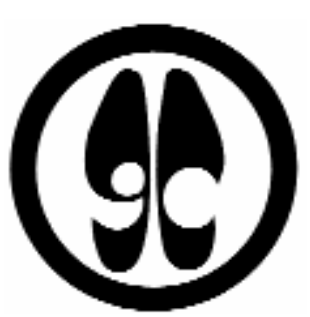

L'allusion à la date commémorative du 9 septembre est occultée par la production symbolisée par une paire de chaussures. À l'origine, la propagande du pouvoir vise notamment à faire accepter une vie ascétique au prodit de la "richesse" de la vie spirituelle. Les années ont démontré un appauvrissement de la culture officielle au profit d'une nette augmentation du niveau de vie matérielle des individus... Le trou dans le pied gauche de la chaussure n'est-il pas alors un clin d'oeil du graphiste, la satire d'une certaine misère spirituelle du pouvoir que les faits contredisent?

81 Mais cette contestation se limite à une attitude plus assimilable à une forme de défense passive qu'à une remise en cause ouverte du système.

82 L'Union des graphistes bulgares brave cependant la censure dans les années 1970, en publiant dans un catalogue professionnel bi-annuel les projets de quelques logos nonretenus pour raison idéologique ${ }^{60} .$.

Night-club de l'hôtel Dobrudja, Albena, 1988.

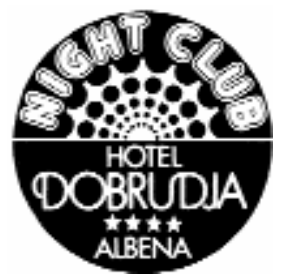

Cet établissement est officiellement ouvert à touts, mais ses prix excessifs le réservent en fait aux Occidentaux (privilégiés par un taux de change monétaire très favorable). Diffusé uniquement à l'intérieur de l'hôtel, bien peu de Bulgares ont donc l'occasion de découvrir ce logo...

Groupe de rock FSB (Formation Studio Balkanton), 1980.

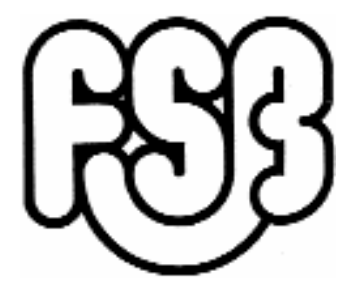


83 Mais il faut donc attendre les années 1980 et l'affaiblissement de l'influence du pouvoir communiste pour voir apparaître une contestation de type offensif de la morale socialiste, avec des noms de marques et un graphisme faisant allusion à des normes proscrites par le régime.

On voit donc surgir, hors de tout contrôle du pouvoir, des thèmes tels que le luxe, l'érotisme, l'imaginaire ou encore l'exotisme suggérés par la marque de jus de fruits Banan, les parfums Dream (écrit en plus en caractères latins!) ou encore Julia (en référence à Roméo et Juliette).

On commence également à créer des logos pour les groupes de rock, les discothèques ou les night-clubs, chose absolument inacceptable par la morale socialiste et inimaginable jusqu'en 1980 ...

Par la reproductibilité et la diffusion de leur expression, les graphistes qui décident dans les années 1980 de passer outre les directives du régime, contribuent donc à exprimer ouvertement l'existence d'une seconde morale dans la société. Ils se font porte-parole de la contestation en mettant à jour certaines contradictions du régime, participant à leur manière à la levée de quelques tabous...

Théâtre du front du travail, Sofia, 1988.

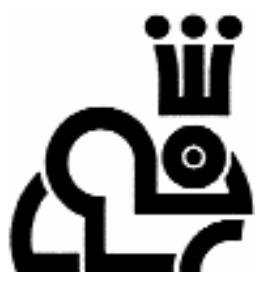

Par sa situation géographique, ce théâtre est plus connu sous le nom officieux de Petit théâtre derrière le canal. Le projet de logo, présenté en 1988, est trop peu adapté au nom officiel du théâtre. Mais il a surtout le defaut, aux yeux des censeurs, de manier, par ses multiples allégories, unr ironie peu appréciée en haut lieu.

N'est-ce pas notamment par le canal que l'on évacue certaines eaux sales... dans lesquelles il faut savoir nager?

Le nom de Petit théâtre derrière le canal ainsi que son logo sont cependant officialisé en 1990, la réalité rattrapant la fiction..

\section{De la propagande à la propagande...}

«Plusieurs propriétés de la marque, comme la structure narrative, sa vocation à produire de la signification et à véhiculer des valeurs, suggèrent que la marque dispose "réellement" d'une capacité à créer des mondes possibles. " ${ }^{61}$

C'est en se fondant sur ce type d'analyse que le pouvoir communiste en Bulgarie a détourné de leurs vocations initiales les marques et les logos afin de les utiliser comme supports et outils de diffusion de son idéologie. Ils sont alors à l'image du pouvoir omnipotent: ils participent à la propagande en se faisant le reflet d'une nouvelle société officielle.

Après 1989, l'ouverture vers une économie de marché fait cependant retrouver aux marques et logos leur rôle de propagande commerciale, au risque de créer artificiellement une nouvelle culture de masse. Il apparaît alors que les marques, dans une économie concurrentielle, utilisent souvent les mêmes techniques (notamment la répétition, la présentation d'un monde idéal pour chacun...) en faisant appel aux 
mêmes pulsions chez les individus que la propagande du pouvoir communiste (pulsions combative, parentale, etc.).

Contrairement à une vision communément diffusée en Occident, l'époque communiste n'est pas une parenthèse dans l'Histoire des pays de l'est car le temps ne s'est arrêté pour personne.

Mais la rupture politique de 1989 a permis, à ceux qui en ont été les sujets, de prendre officiellement leurs distances avec la propagande du pouvoir socialiste. L'étude des œuvres des graphistes amène alors à se poser les questions primordiales de leurs enjeux et donc de l'implication, volontaire ou imposée, de chaque individu dans sa société...

\section{NOTES}

1. Schaal (Jean-François), Le Pouvoir, Paris : Ellipses, Paris, 1994, p. 181.

2. Précisons cependant que l'UFD (coalition de plusieurs mouvements politiques bulgares créée en 1990 en opposition au Parti communiste) a finalement rejeté les propositions de Jacques Séguéla, plus empressé de présenter un devis exorbitant que de chercher à comprendre la situation de la Bulgarie.

3. Bongrand (Michel), Le Marketing politique, Paris : Presses universitaires de France, 1993, p. 14.

4. Domenach (Jean-Marie), La Propagande politique, Paris : Presses universitaires de France, 1979, p. 8.

5. Ibid., p. 16.

6. Tchakhotine (Serge), Le Viol des foules par la propagande politique, Paris : Gallimard, 1992, p. 129.

7. Domenach (Jean-Marie), op.cit., p. 17.

8. Machiavel (Niccolo), Le Prince, Paris : Fernand Nathan, 1984, p. 115.

9. Domenach (Jean-Marie), op.cit., p. 5.

10. Driencourt (Jacques), La Propagande, nouvelle force politique, Paris : Librairie Armand Colin, 1950 , p. 79.

11. Ibid., p. 1. Il est stupéfiant de constater à quel point l'immense majorité des ouvrages français traitant de la propagande dans l'Europe du $\mathrm{XX}^{\mathrm{ème}}$ siècle ne décelle la manipulation que dans les régimes nazis et communistes, rejetant de fait l'idée que la manipulation à grande échelle puisse toucher les auteurs eux-mêmes.

12. Driencourt (Jacques), op.cit., p. 105.

13. Lénine (Vladimir Ilitch), Que faire? Les questions brûlantes de notre mouvement, Paris : Éditions Sociales / Moscou : Éditions du Progrès, 1979, pp. 145-146.

14. Schnitzer (Luda et Jean), Histoire du cinéma soviétique, 1919-1940, Paris : Pygmalion, 1979, p. 13.

15. Domenach (Jean-Marie), op.cit., p. 47.

16. Prenons pour exemples la chanson d'un film (Lili Marlene, utilisée dans l'armée allemande pendant la Seconde Guerre mondiale) ou le portrait d'un dirigeant dans un livre (le portrait de Mao Zedong dans de nombreux ouvrages politiques édités en Chine dans les années 1960-1970). Citons également la conférence de presse du Front de libération nationale de la Corse (FLNC), 
dans la nuit du 11 au 12 janvier 1996 à Tralonca, dont le message résidait apparemment tout autant dans la mise en scène bien étudiée (atmosphère de clandestinité, détermination, cohésion, menace par la participation de centaines de personnes en tenue militaire et équipées de matériel de combat moderne, ...) que dans les informations transmises oralement.

17. Date officielle de la prise du pouvoir par les communistes.

18. Arendt (Hannah), Le Système totalitaire, Paris : Seuil, 1972, p. 80.

19. Nietzsche (Friedrich), La Volonté de puissance (tome II), Paris : Gallimard, 1995, p. 234.

20. Volkoff (Vladimir), La Désinformation arme de guerre, Paris : Julliard / L'Âge d'Homme, 1986, p. 245.

21. Schaal (Jean-François), op.cit., p. 166.

22. Notons toutefois l'exceptionnel logo de Bulgarkonserv, ayant étrangement échappé à la règle en 1964 en utilisant la représentation d'une femme.

23. Dimitar Blagoev est le fondateur de l'ancêtre du Parti Communiste Bulgare en 1892.

24. Vassil Kolarov est le compagnon de lutte de Gueorgui Dimitrov.

25. Semprini (Andrea), La Marque, Paris : Presses universitaires de France, 1995, p. 36.

26. Gourevitch (Jean-Paul), La Propagande dans tous ses états, Paris : Flammarion, 1981, p. 70.

27. Orwell (George), 1984, Paris : Gallimard, 1990, pp. 62-63.

28. Fejtö (François), Histoire des démocraties populaires. 2. Après Staline, Paris : Seuil, 1992, p. 206.

29. Tchakhotine (Serge), op.cit., p. 51.

30. Ibid., p. 53.

31. Dollot (Louis), Culture individuelle et culture de masse, Paris : Presses universitaires de France, 1993, p. 80.

32. Huxley (Aldous), Le Meilleur des mondes, Paris : Presses pocket, 1990, p. 248.

33. Reich (Wilhelm), La Psychologie de masse du fascisme, Paris : Payot, 1972, pp. 40-41.

34. Chef de l'État bulgare de 1956 à 1989.

35. Domenach (Jean-Marie), op.cit., p. 89.

36. Un enfant maltraité par ses parents, par exemple, n'abandonne pas pour autant un sentiment d'amour filial.

37. Domenach (Jean-Marie), op.cit., p. 88.

38. Voir à ce sujet les expériences sur l'obéissance menées par Stanley Milgram, aux cours desquelles un individu présenté comme "normal" accepte, sous la direction d'un "moniteur", de se livrer à la torture par l'électricité sur une autre personne. Stanley Milgram constate entre autres que «le processus d'adaptation de pensée le plus courant chez le sujet obéissant est cet abandon de toute responsabilité personnelle; il attribue l'entière initiative de ses actes à l'expérimentateur qui représente une autorité légitime. Il ne se voit pas du tout en être humain assumant pleinement sa conduite, mais en instrument aux mains d'une autorité étrangère.» (Milgram (Stanley), Soumission à l'autorité, un point de vue expérimental, Paris : Éditions CalmannLévy, 1986, p. 24).

39. Hobbes (Thomas), Léviathan, traité de la matière, de la forme et du pouvoir de la république ecclésiastique et civile, Paris : Éditions Sirey, 1971, p. 166.

40. L'influence grandissante des anarchistes bulgares (dès les années 1920) est ressentie par les staliniens comme un facteur pouvant destabiliser leur pouvoir. On comprend alors pourquoi la FACB est l'une des premières cibles du nouveau régime, même si le pouvoir communiste n'a jamais vraiment réussi à l'éliminer.

41. Les Bulgares parlent au monde, Commission d'aide aux antifascistes de Bulgarie, Paris, 1949, pp. 29-30.

42. Gueorgui Dimitrov est mort le 2 juillet 1949.

43. Ludmila Jivkova, ministre de la Culture, est la fille du chef de l'État.

44. Kadaré (Ismaiil), La Légende des légendes, Paris : Flammarion, 1995, p. 157.

45. Gourevitch (Jean-Paul), op.cit., p. 102. 
46. Tchakhotine (Serge), op.cit., pp. 281-282.

47. Gourevitch (Jean-Paul), op.cit., p. 106.

48. Pravetz est la ville natale de Todor Jivkov alors au pouvoir.

49. Chaîne de montagnes fortement liée aux mouvements nationalistes macédoniens.

50. Lieu de la bataille qui permet aux Russes de traverser le pays pour contribuer à l'indépendance de 1878.

51. Burdeau (Georges), « Nation », in Encyclopcedia Universalis, Paris : Encyclopædia Universalis France, 1989, tome 16, p. 5.

52. Sfez (Lucien), La Symbolique politique, Paris : Presses universitaires de France, 1988, p. 85.

53. Girardet (Raoul), «Nation», in Encyclopcedia Universalis, Paris : Encyclopædia Universalis France, 1989, tome 16, p. 18.

54. Les citoyens de culture turque représentent environ $10 \%$ de la population de la Bulgarie.

55. Staline (Joseph), Euvres (tome 3), Paris : Éditions sociales, 1954, p. 58.

56. Kostov (Vladimir), "Bulgarie ", in Encyclopcedia Universalis (tome 4), Paris : Encyclopædia Universalis France, 1989, p. 648.

57. Burdeau (Georges), art.cit., p. 6.

58. La Dobroudja est une région frontalière avec la Roumanie dont l'appartenance a été maintes fois contestée.

59. Conte (Francis), Les Slaves, aux origines des civilisations d'Europe centrale et orientale, Paris: Albin Michel, 1986, p. 614.

60. Catalogue connu sous le nom de Bulletin de l'Union des peintres bulgares, section Graphisme, Sofia : Éditions Bulgarski houdojnik.

61. Semprini (Andrea), op.cit., p. 75.

\section{RÉSUMÉS}

La politique étant un "produit » comparable à une marchandise, les logos ont suivi le même développement que les techniques de propagande politique. Ils font partie intégrante de l'idéologie en vigueur. C'est ce que tente de montrer l'auteur avec l'exemple de la Bulgarie, où ce n'est qu'à la fin des années 80 qu'une contestation s'exprime au moyen de noms de marques et d'un graphisme faisant allusion à des normes proscrites par le régime.

\section{AUTEUR}

\section{MARIA TZVETKOVA}

Diplômée de l'Institut supérieur des beaux-arts de Sofia et de l'École nationale supérieure des arts décoratifs de Paris, Maria Tzvetkova est graphiste professionnelle. 